\title{
Nuclear Masses in Astrophysics
}

\author{
Christine Weber ${ }^{a}$, Klaus Blaum $^{* b}$, and Hendrik Schatz ${ }^{c}$ \\ ${ }^{a}$ Department of Physics, University of Jyväskylä, FI-40014 Jyväskylä, Finland \\ ${ }^{b}$ Max-Planck-Institut für Kernphysik, D-69117 Heidelberg, Germany \\ ${ }^{c}$ NSCL, Michigan State University, East Lansing, MI 48824-1321, USA \\ E-mail: christine.weber@jyu.fi, klaus.blaum@mpi-hd.mpg.de, \\ schatz@nscl.msu.edu
}

\begin{abstract}
Among all nuclear ground-state properties, atomic masses are highly specific for each particular combination of $N$ and $Z$ and the data obtained apply to a variety of physics topics. One of the most crucial questions to be addressed in mass spectrometry of unstable radionuclides is the one of understanding the processes of element formation in the Universe. To this end, accurate atomic mass values of a large number of exotic nuclei participating in nucleosynthesis are among the key input data in large-scale reaction network calculations. In this paper, a review on the latest achievements in mass spectrometry for nuclear astrophysics is given.
\end{abstract}

10th Symposium on Nuclei in the Cosmos

July 27 - August 12008

Mackinac Island, Michigan, USA

${ }^{*}$ Speaker. 


\section{Introduction}

To date mainly two experimental approaches for high-precision mass measurements on shortlived nuclides with the required relative mass uncertainties of $10^{-7}$ exist, namely storage ring and Penning trap mass spectrometry [1]. The installation of such ion-storage devices at on-line facilities allows to combine the advantages of extremely sensitive, high-precision experiments for studies on a vast number of exotic nuclides that are readily provided today. Beams far off the valley of $\beta$ stability are available at accelerator facilities such as ISOLDE/CERN, GSI in Darmstadt, JYFL in Jyväskylä, or the NSCL at Michigan State University. They are either produced in proton-induced spallation, fragmentation, or fission reactions in a thick target (ISOL approach) or via fusion-evaporation or fragmentation and a subsequent in-flight separation. Depending on the particular production scheme, several initial steps including laser- or surface-ionization techniques [2] in the ISOL approach, or fast stopping and extraction schemes are employed [3, 4].

Whereas neutron-deficient nuclides close to the $N=Z$ line and in the vicinity of the rapid proton capture $(r p)$-process are routinely produced and measurements even beyond the proton drip line have been successfully conducted farther up around $A=145$ [5], neutron-rich nuclides studied are located close to the $N=50$ shell closure $[6,7]$ or span from the nickel $(A=73)$ to the palladium $(A=120)$ isotopes $[8,9,10,11,12]$. However, the presumed path of the $r$-process is merely reached by high precision mass measurements in the region around $N=50$ and close to $N=82$ within the tin isotopes [13]. ${ }^{130} \mathrm{Cd}$ has been reached with the less precise $\beta$-endpoint method [14].

\section{Mass spectrometry of short-lived isotopes}

\subsection{Penning traps}

At present six Penning trap facilities are operational at accelerators. These experimental setups include ISOLTRAP [15] at ISOLDE/CERN, the Canadian Penning trap (CPT) [16] at the Argonne National Laboratory ANL, SHIPTRAP [17] at GSI/Darmstadt, the JYFLTRAP facility [18] in Jyväskylä, LEBIT [19] at NSCL(MSU), and TITAN [20] at TRIUMF/Vancouver. The first Penning trap mass spectrometer at a nuclear reactor is presently in the commissioning phase at TRIGA-Mainz [21]. The common pre-requisite in all experiments is an ion deceleration, accumulation, and cooling as successfully obtained in linear radiofrequency quadrupole (rfq) coolers and bunchers $[22,23,24]$ with the goal to deliver ion pulses with a well-defined emittance to the subsequent Penning trap setups.

Figure 1 shows two geometric configurations of a Penning trap as employed in mass spectrometry on short-lived nuclides. It is operated in a combination of a high-field $(B \sim 7 \mathrm{~T})$, highhomogeneity $\left(\Delta B / B \leq 10^{-6} / \mathrm{cm}^{3}\right)$ superconducting magnet and a quadrupolar electrostatic storage potential that is applied as a voltage difference $V_{0}$ between the ring electrode and both endcaps of the trap (see Fig. 1). The resulting ion motions in the combined fields are depicted in the right part of the figure. They consist of a superposition of three ideally independent harmonic oscillations with characteristic eigenfrequencies $\omega$ : an axial motion $\left(\omega_{z}\right)$ and two radial motions, the modified cyclotron motion $\left(\omega_{+}\right)$and a slow $\vec{E} \times \vec{B}$ drift, the magnetron motion $\left(\omega_{-}\right)$. Their frequencies are

$$
\omega_{z}=\sqrt{\frac{q V_{0}}{m d^{2}}} \quad \text { and } \quad \omega_{ \pm}=\frac{\omega_{c}}{2} \pm \sqrt{\frac{\omega_{c}^{2}}{4}-\frac{\omega_{z}^{2}}{2}},
$$


where $\omega_{c}=\frac{q}{m} B$ is the free cyclotron frequency and $d$ the characteristic trap dimension [25]. The sum frequency of both radial motions in an ideal Penning trap $\omega_{+}+\omega_{-}=q B / m$ is directly related to the charge-to-mass ratio $q / m$. It is determined in a time-of-flight detection method [26]. Here, ions are released from the trap and their flight time to a detector in the magnet's fringe field is recorded. Prior to their ejection, ions are excited with an azimuthal quadrupolar RF field around $\omega_{c}$ [27]. In case of a resonant excitation at $\omega_{c}$ the ion's radial kinetic energy is maximized, which is transformed into an additional axial acceleration while ions travel along the magnetic field gradient. Atomic masses $m$ are derived using alternating calibration measurements of nuclides with precisely known mass values or close-by carbon cluster ions ${ }^{12} \mathrm{C}_{n}^{+}$for absolute mass calibration $[28,29]$. In this way, relative mass uncertainties $\delta \mathrm{m} / \mathrm{m}$ as low as few times $10^{-8}$ are routinely achieved.
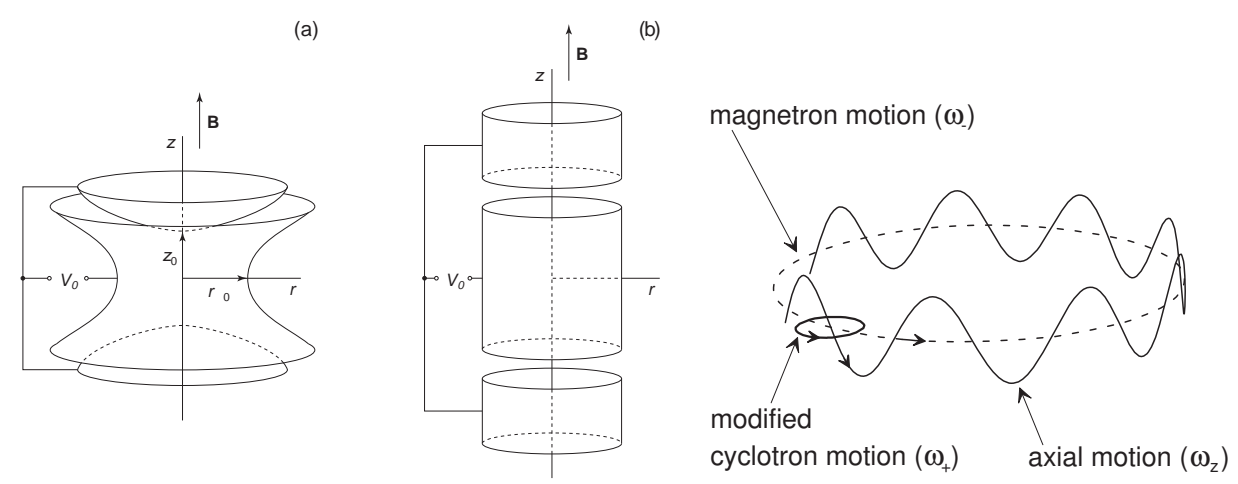

Figure 1: Left: Penning trap configurations. A trap consists of a central ring electrode and two endcap electrodes. A hyperbolical electrode geometry (a) generates the quadrupolar shape of the electrostatic storage potential. Traps with a cylindrical electrode geometry (b) are frequently used due to easier capture properties. Right: Schematic of the characteristic eigenmotions of a charged particle in a Penning trap.

\subsection{Ion storage rings}

A large-scale approach for mass spectroscopy of stored ions are direct measurements in storage rings. At present, such measurements are routinely performed at the GSI Experimental Storage Ring ESR [30], and are being started at the CSRe storage ring in IMP Lanzhou [31]. Mass measurements are also planned at RIKEN with a new RI-RING project [32]. At GSI, exotic nuclides are produced in projectile-fragmentation reactions or fission in thin targets, selected in the Fragment Separator (FRS) and are subsequently injected into the ESR. Figure 2 shows a schematic layout of the ESR storage ring. For ions circulating in the ring the relative difference in the mass-to-charge ratio $m / q$ of the revolving ion species is expressed as

$$
\frac{\Delta f}{f}=-\frac{1}{\gamma_{t}^{2}} \frac{\Delta(m / q)}{m / q}+\left(1-\frac{\gamma^{2}}{\gamma_{t}^{2}}\right) \frac{\Delta v}{v}
$$

where $\Delta f / f$ is the relative difference in the revolution frequency, $\gamma$ is the Lorentz factor, and $v$ is the velocity.

Two different approaches exist for a direct determination of $m / q$ : for rather long-lived nuclides with half-lives of more than a few seconds ions can be cooled in the electron cooler of the ring with the aim to reduce their relative velocity spread $(\Delta v / v)$ to few $10^{-7}$. Hence, the term on the right in Eq. (2.2) is negligible and the revolution frequencies directly correspond to $m / q$. Here, 


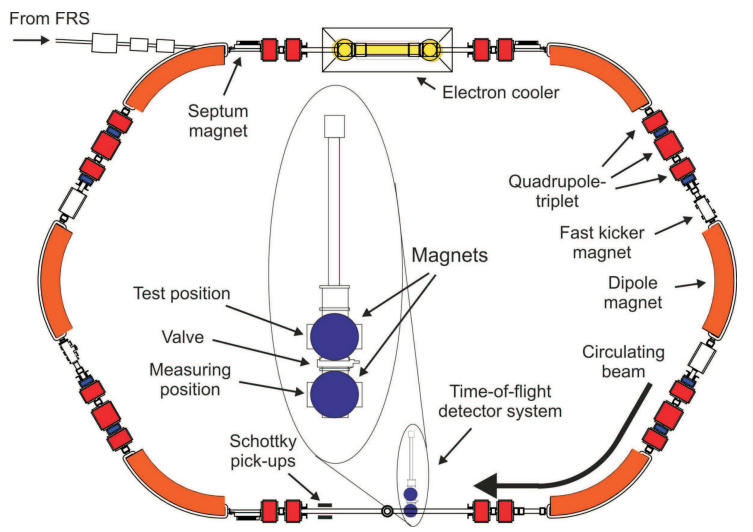

Figure 2: (Color online) Layout of the Experimental Storage Ring (ESR) at GSI/Darmstadt. The circumference of the ring is $108 \mathrm{~m}$. Ions are injected from the Fragment Separator (FRS). In experiments with the Schottky technique, ions are cooled in the electron cooler and their relative velocity spread is reduced by five orders of magnitude. Induced signals of revolving ions are detected at the Schottky pick-up electrodes. Experiments in the isochronous operation mode register the revolution times of circulating ions in a dedicated time-of-flight detector system.

induced signals are recorded in the Schottky pick-up electrodes and are subsequently Fourier analyzed. A multitude of ion species is stored simultaneously in the ring and hence, the masses of several nuclides can be determined in an extremely efficient way, whereas those ions with precisely known masses are used for calibration. Since multiply charged ions are detected, single-ion sensitivity is reached in these measurements. Moreover, the identification of ions in different charge states is useful as a check for internal consistency of the obtained data set. Typical relative mass uncertainties obtained are few times $10^{-7}$, corresponding to $\delta m \approx 30 \mathrm{keV}$ for $A \approx 90-200$ [33].

Alternatively, the so-called isochronous operation mode of the storage ring is employed [34]. Here, the Lorentz factor $\gamma$ is selected to be equal to the transition energy $\gamma_{t}$ and again, the second term in Eq. (2.2) vanishes such that revolution frequencies are proportional to $m / q$. This method does not require electron cooling and is well-suited for nuclides with very short half-lives as low as a few ten $\mu$ s [35]. In this case a dedicated time-of-flight detector is used to measure the revolution times of stored ions [36]. In recent studies the masses of several neutron-rich fission fragments were determined using an additional $B \rho$ determination prior to the injection into the ring and typical mass uncertainties of about $\delta m \approx 120 \mathrm{keV}$ were obtained [37].

\section{Neutron-deficient nuclei and implications for $r p$ - and $v p$-process nucleosynthesis}

A large set of new data on neutron-deficient nuclei relevant for studies of the astrophysical $r p$-process $[38,39]$ and the recently proposed $v p$-process $[40,41]$ were obtained with Penning trap mass spectrometers world-wide. They support the modelling of these nucleosynthesis pathways, which aims at understanding the final elemental abundances and energy production, and at comparing model results with the growing number of astronomical observations in a quantitative way. The $r p$-process in type I x-ray bursts [39] starts, for example, from a breakout of the hot CNO cycle by a sequence of $\alpha$-induced reactions and proceeds then via rapid proton-capture reactions and subsequent $\beta^{+}$decays close to $N=Z$. In particularly hydrogen rich bursts it can reach up to 
tellurium, where the predominant $\alpha$ instability, ${ }^{107} \mathrm{Te}$ and ${ }^{108} \mathrm{Te}$, returns the flow into the closed SnSbTe cycles [42].

In addition to $\beta$-decay half-lives, precise atomic mass data are among the most critical nuclear parameters in reaction network calculations for nucleosynthesis. The detailed reaction flow is determined by individual mass differences, the single-proton separation energies, moreover mass values are used to calculate reaction energies to model capture processes. To this end, mass uncertainties $\delta m$ on the order of less than $10 \mathrm{keV}$, i.e. $\delta m / m \leq 10^{-7}$, are required [43], which are routinely achieved in precision Penning trap mass spectrometry.

In the lower part of the $r p$-process, direct mass measurements of the bare short-lived ${ }^{44} \mathrm{~V}$, ${ }^{48} \mathrm{Mn},{ }^{41} \mathrm{Ti}$, and ${ }^{45} \mathrm{Cr}$ ions where obtained in isochronous mass spectrometry (IMS) at the ESR storage ring [35]. The mass uncertainties of few $100 \mathrm{keV}$ obtained in these studies contribute with first data on proton separation energies beyond scandium and the $r p$-process flow through the titanium to manganese isotopes is discussed.

Along the $r p$-path, the so-called waiting point nuclei, ${ }^{64} \mathrm{Ge},{ }^{68} \mathrm{Se},{ }^{72} \mathrm{Kr}$, and ${ }^{76} \mathrm{Sr}$, are of particular importance, since they can cause a delay in the process. At a waiting point, a $(p, \gamma)-(\gamma, p)$ equilibrium is reached between proton-capture and photodisintegration reactions while further proton captures are hindered by low $Q$ values to the next, proton-unbound isotone. The process slows down until these nuclides undergo $\beta$ decay. The effective lifetime of a waiting point can be as long as its $\beta$-decay half-life, modified by the lifetime against possible two-proton captures [43]. Studies of these nuclei and their close vicinity are needed to constrain the limits on the effective lifetimes, which depend exponentially on the $Q$ value for proton capture. Some of the critical masses around ${ }^{64} \mathrm{Ge}$ and ${ }^{68} \mathrm{Se}$ have been both determined with CPT [44, 45] and LEBIT [46], respectively, reducing the uncertainties in the effective lifetimes. Measurements on ${ }^{72-74} \mathrm{Kr}$ were obtained with ISOLTRAP [47]. The latter study indicates that the $\beta$-decay lifetime is modified by less than $20 \%$. In addition, several nuclides such as neutron-deficient strontium isotopes up to the waiting point ${ }^{76} \mathrm{Sr}$ [48], or neutron-deficient isotopes of selenium and bromine [49] were studied at ISOLTRAP.

Across the higher-mass region of the $r p$-process path above $A=80$ broad mass determinations have been performed with JYFLTRAP, SHIPTRAP and CPT. Figure 3 gives an overview on all nuclides studied in either of the first two experiments. A possible pathway of the $r p$-process for steady-state burning (from Schatz [42], solid lines) is shown together with a possible path of the $v p$ process (dashed lines). The data amount to about 75 newly determined mass values which are not included in the published issue of the Atomic Mass Evaluation 2003 [50]. Their mass uncertainties $\delta m$ are well below the wanted limit of $10 \mathrm{keV}$ as required in nucleosynthesis calculations [43].

Masses of yttrium, zirconium, and niobium isotopes were determined at JYFLTRAP employing heavy-ion induced ${ }^{32} \mathrm{~S}+\left({ }^{54} \mathrm{Fe}\right.$ or $\left.{ }^{\text {nat }} \mathrm{Ni}\right)$ reactions [51] with resulting typical uncertainties of $7 \mathrm{keV}$. In a common approach of JYFLTRAP and SHIPTRAP the masses of 21 further nuclides up to and including ${ }^{96} \mathrm{Pd}$ were studied [52], and almost half of them were determined experimentally for the first time. The results of both Penning trap experiments are in excellent agreement with each other, and a weighted mean with an improved final uncertainty as low as $2.9 \mathrm{keV}$ was given. Results for the most exotic isotopes deviate substantially from data in AME2003, which are mostly stemming from $\beta$-endpoint measurements and extrapolations of systematic trends. The impact of the new results was studied in nucleosynthesis calculations of the $v p$-process. Detailed reaction flow pattern were compared with calculations that only include the data of AME2003. Since the new 


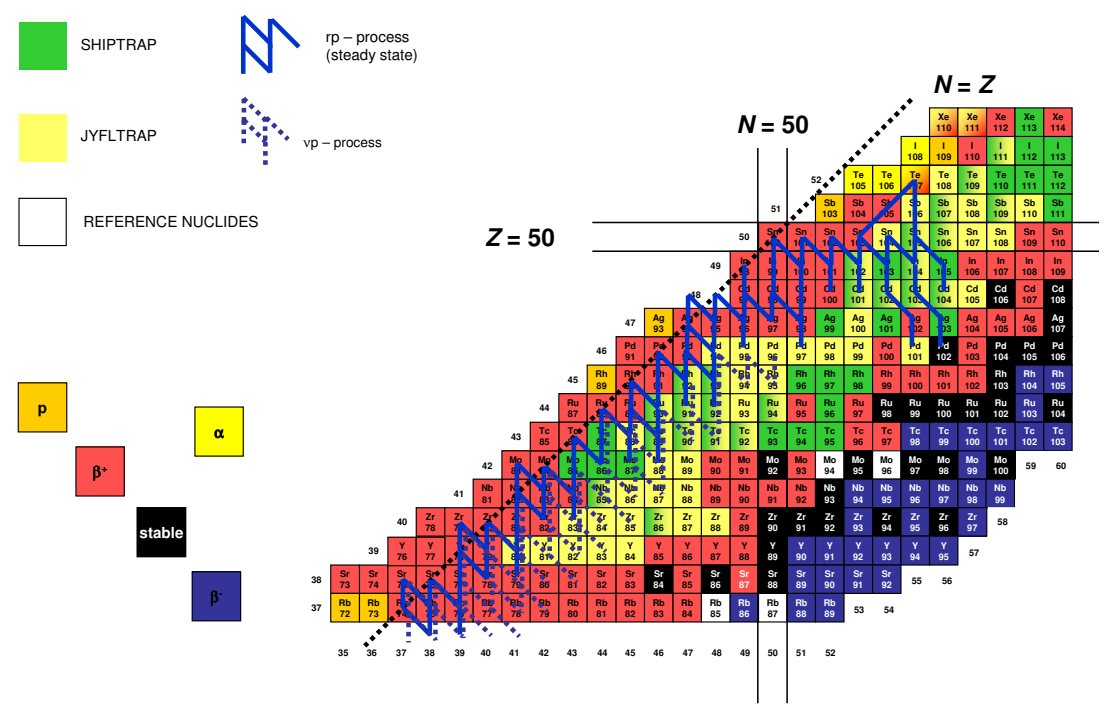

Figure 3: (Color online) Section of the nuclear chart with the upper region of the $r p$ - and $v p$-process pathways. Nuclides studied with the JYFLTRAP (yellow) or the SHIPTRAP (green) mass spectrometer are indicated. For a detailed review see text.

mass values, for example the one of ${ }^{88} \mathrm{Tc}$ results in a proton-separation energy which is $1 \mathrm{MeV}$ smaller than in the AME2003 systematics, the reaction flow around ${ }^{88} \mathrm{Tc}$ is strongly modified. However, the final abundances for the $v p$-process calculations were found to be almost unchanged.

Nuclides above ${ }^{96} \mathrm{Pd}$ were investigated in independent experiments at either SHIPTRAP [53] or JYFLTRAP [54]. Whereas heavy-ion induced fusion reactions of ${ }^{58} \mathrm{Cr}$ and ${ }^{58} \mathrm{Ni}$ beams on ${ }^{58} \mathrm{Ni}$ targets were employed for measurements at SHIPTRAP, measurements in Jyväskylä focused on the verification of production possibilities of these nuclides in light-ion induced reactions with $p$ or ${ }^{3} \mathrm{He}$ beams. Among the nuclides studied only the most neutron-deficient ones are relevant for $r p$-process nucleosynthesis. Here, discrepancies of up to $2 \sigma$ are observed in the cadmium isotopes ${ }^{101,102,104} \mathrm{Cd}$. However, this apparent discrepancy can possibly be solved by new data from ISOLTRAP along the cadmium isotopic chain ${ }^{99-109} \mathrm{Cd}$ [55]. Most recently, the mass values of the exotic ${ }^{85} \mathrm{Mo}$ and ${ }^{87} \mathrm{Tc}$ nuclides were determined for the first time in experiments at SHIPTRAP and the mass values of ${ }^{86,87} \mathrm{Mo},{ }^{93-95} \mathrm{Tc},{ }^{94,96} \mathrm{Ru}$, and ${ }^{96-98} \mathrm{Rh}$ were measured and substantially improved [56].

The mass values of several nuclides from molybdenum to rhodium were determined with the CPT $[57,58,59]$. As the production of the light $p$ nuclei, ${ }^{92,94} \mathrm{Mo}$ and ${ }^{96,98} \mathrm{Ru}$ is not quantitatively understood, i.e. these nuclides are observed in the Solar System in greater abundance than predicted in $p$-process theory, the latter publications focus on the inconsistency in the molybdenum isotopes. The $v p$-process was introduced to explain the creation of these nuclides in proton-rich ejecta of supernova explosions in neutrino-driven winds [40, 41]. It was shown that to reproduce the observed abundance ratios, current models would require a separation energy $S_{p}$ of ${ }^{93} \mathrm{Rh}$, calculated as $-M\left({ }^{93} \mathrm{Rh}\right)+M\left({ }^{92} \mathrm{Ru}\right)+M\left({ }^{1} \mathrm{H}\right)$, of $1.64 \pm 0.1 \mathrm{MeV}[60,61]$. However, all Penning trap experiments and the estimation in AME [50] yield a separation energy that differs considerably from this value. This discrepancy might indicate either the presence of a different production site, 
or deficiencies in the astrophysical models. Though there is an agreement in the $S_{p}$ value between the trap data, the mass values of both nuclides involved, ${ }^{93} \mathrm{Rh}$ and ${ }^{92} \mathrm{Ru}$, deviate by about $1.5 \sigma$ in measurements from SHIPTRAP, JYFLTRAP [52] and the ones of the Canadian Penning trap [58].

In the endpoint region of the $r p$-process the mass values of thirteen nuclides were determined at JYFLTRAP (see Fig. 3). The resulting one-proton separation energies in the studied antimony isotopes are particularly relevant in order to estimate the fraction of proton captures that flow into the closed SnSbTe cycles. With a new value of the proton separation energy $S_{p}$ for ${ }^{105} \mathrm{Sb}$, determined indirectly from the $\alpha$ energy of ${ }^{109}$ I in Ref. [62], the formation of a significant cycle at ${ }^{104} \mathrm{Sn}$ is excluded. The new data in this region, in particular the first experimental determinations on ${ }^{106} \mathrm{Sb},{ }^{108} \mathrm{Sb}$, and ${ }^{110} \mathrm{Sb}$ test the concept of the $r p$ endpoint and explore a possible leakage past the closed SnSbTe cycles [63].

\section{Neutron-rich nuclei and implications for $r$-process nucleosynthesis}

The rapid neutron-capture process ( $r$-process) is responsible for the origin of about half of the heavy elements beyond germanium in the cosmos. Elements such as europium, gold, platinum, or uranium are mainly produced in the $r$-process $[64,65]$. However, where this process occurs is not known with certainty and its understanding is one of the greatest challenges of modern nuclear astrophysics. The isotopic abundance pattern not only depends on the chosen astrophysical environment, e.g. the neutron density, but also sensitively on the underlying nuclear physics processes and parameters $[66,67]$. Thus, accurate mass data on the extremely neutron-rich nuclei participating in the $r$-process are of utmost importance to compare the signature of specific models with astronomical data now becoming available from observations of metal-poor stars in the Galaxy [68].

Recently, the TOF-B $\rho$ technique that includes a position measurement for magnetic rigidity correction has been implemented at the NSCL facility using the A1900 separator and the S800 spectrograph. The first experiment, focused on the neutron-rich isotopes in the region of $Z \sim 20$ 30 , important for r-process calculations as well as for calculations of processes occurring in the crust of accreting neutron stars, has been successfully performed [69, 70].

A number of neutron-rich nuclei close to the $r$-process path have been studied with the IMS technique at the ESR [37]. The experimental mass values for iodine isotopes including the newly determined ones are compared to predictions of several modern theories in Fig. 4. It is clear to see that the ETFSI-Q model has a divergent trend beyond $N=80$ and fails to describe the new mass data. The models in this figure span from macroscopic-microscopic to self-consistent microscopic models, which predict very different structure of $r$-process nuclides. Calculations of reaction pathways based on these models yield abundances which sometimes differ by several orders of magnitude [71]. It is therefore essential to provide the experimental basis for testing these predictions and consequently improving the underlying models.

Most modern $r$-process models predict that the $r$-process occurs at high temperatures of a billion degrees or more. At such high temperatures energetic photons can excite nuclei in a way that they emit neutrons. These so-called photodisintegration reactions can counteract the rapid neutron captures. At which nucleus this happens within an isotopic chain depends mainly on the 


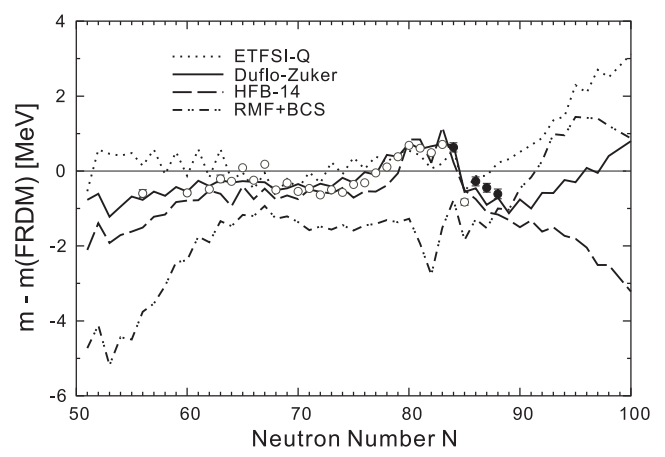

Figure 4: Mass comparison of model predictions and experimental data for iodine isotopes (from Ref. [37]).

binding energy of the neutrons in the nuclei and therefore on the nuclear masses. In this context $r$ process waiting points, i.e. nuclides where photodisintegration wins over neutron capture and thus the $r$-process temporarily stops and waits for the $\beta$ decay into the next isotopic chain, are of highest importance and their masses can be provided with relative uncertainties as low as $10^{-7}$. Nuclear masses therefore largely determine the path of the $r$-process on the chart of nuclides. Together with the $\beta$-decay half-lives of the waiting point nuclei, the masses determine also the speed of the process and the final abundance pattern. The most influential waiting points in the $r$-process path are believed to be ${ }^{80} \mathrm{Zn},{ }^{130} \mathrm{Cd}$, and ${ }^{195} \mathrm{Tm}$, responsible for producing the pronounced abundance peaks observed around mass numbers $A$ equals 80, 130, and 195, respectively (see Ref. [65]).

To date, $Q$-value measurements related to these waiting points were only performed using the $\beta$-endpoint technique in the case of ${ }^{130} \mathrm{Cd}$ with a mass uncertainty of $150 \mathrm{keV}$ [14] and very recently using precision Penning trap mass spectrometry in the case of ${ }^{80} \mathrm{Zn}$ with an uncertainty of only a few $\mathrm{keV}[6,7]$. While the precision achieved with the $\beta$-endpoint technique is not sufficient to perform reliable $r$-process calculations for particular key nuclides, the extremely low uncertainty meanwhile routinely achievable with Penning trap mass measurements allowed, in the case of ${ }^{80} \mathrm{Zn}$, to extract a well-defined map of conditions for a major $r$-process waiting point to be on the reaction path.

\section{Conclusions and future perspectives}

By the huge amount of improved mass data available, a more reliable modelling of the astrophysical nucleosynthesis processes is feasible. New network calculations should ideally comprise up-to-date data from different experiments to provide best possible mass values including extrapolations. Together with better defined conditions within the actual astrophysical sites, these enable to answer detailed questions on both sides of the valley of $\beta$ stability.

Unfortunately, most of the extremely neutron-rich nuclei in the $r$-process are still beyond the reach of existing Nuclear Physics accelerator facilities since they have very small production rates. However, upgrades toward future radioactive beam (RIB) facilities such as the future facility FAIR at GSI in Darmstadt, Germany [72], the BigRIPS separator at RIKEN/Japan [73] or a new advanced rare-isotope accelerator in the US [74], aim at higher beam intensities and the production of neutron-rich nuclides farther away from stability and may overcome this limitation. For exam- 
ple, the future ILIMA@FAIR project will enable to address nuclei with production rates as low as 1 ion per day/week, corresponding to production rates on the order of picobarn [37].

The authors highly appreciate the detailed comments to the manuscript from Yuri A. Litvinov. Financial support by the HGF (contract VH-NG-037), by the German BMBF (contract 06MZ215), by the EU within NIPNET (contract HPRI-CT-2001-50034), Ion Catcher (contract HPRI-CT-200150022), EURONS (JRAs TRAPSPEC and DLEP), the Academy of Finland under the Centre of Excellence Programmes 2000-2005 (Nuclear and Condensed Matter Physics Programme), 20062011 (Nuclear and Accelerator Based Physics Programme at JYFL), and NSF grant numbers PHY0606007 and PHY-02-16783 (Joint Institute for Nuclear Astrophysics) is gratefully acknowledged.

\section{References}

[1] K. Blaum, Phys. Rep. 425 (2006) 1.

[2] U. Köster, Nucl. Phys. A 701 (2002) 441c.

[3] J. Äystö, Nucl. Phys. A 693 (2001) 477.

[4] G. Bollen, D. J. Morrissey, and S. Schwarz, Nucl. Instr. and Meth. A 550 (2005) 27.

[5] C. Rauth et al., Phys. Rev. Lett. 100 (2008) 012501.

[6] J. Hakala et al., Phys. Rev. Lett. 101 (2008) 052502.

[7] S. Baruah et al., accepted for publication in Phys. Rev. Lett. (2008), arXiv:0811.2294.

[8] S. Rahaman et al., Eur. Phys. J. A 34 (2007) 5.

[9] S. Rahaman et al., Eur. Phys. J. A 32 (2007) 87.

[10] P. Delahaye et al., Phys. Rev. C 74 (2006) 034331.

[11] U. Hager et al., Nucl. Phys. A 793 (2007) 20.

[12] U. Hager et al., Phys. Rev. C 75 (2007) 064302.

[13] M. Dworschak et al., Phys. Rev. Lett. 100 (2008) 072501.

[14] I. Dillmann et al., Phys. Rev. Lett. 91 (2003) 162503.

[15] M. Mukherjee et al., Eur. Phys. J. A 35 (2008) 1.

[16] G. Savard et al., Int. J. of Mass Spectrom. 251 (2006) 252.

[17] M. Block et al., Eur. Phys. J. A 25 S01 (2005) 49.

[18] V. S. Kolhinen et al., Nucl. Instr. and Meth. B 528 (2004) 776.

[19] R. Ringle et al., Int. J. of Mass Spectrom. 251 (2006) 300.

[20] J. Dilling et al., Int. J. of Mass Spectrom. 251 (2006) 198.

[21] J. Ketelaer et al., Nucl. Instr. and Meth. A 594 (2008) 162.

[22] A. Nieminen et al., Nucl. Instr. and Meth. A 469 (2001) 244.

[23] F. Herfurth et al., Nucl. Instr. and Meth. A 469 (2001) 254.

[24] S. Schwarz et al., Nucl. Instr. and Meth. B 204 (2003) 474.

[25] L. S. Brown and G. Gabrielse, Rev. Mod. Phys. 58 (1986) 233.

[26] G. Gräff, H. Kalinowsky, and J. Traut, Z. Phys. A 297 (1980) 35.

[27] M. König et al., Int. J. Mass. Spectrom. Ion Process. 142 (1995) 95.

[28] K. Blaum et al., Eur. Phys. J. A 15 (2002) 245.

[29] A. Kellerbauer et al., Eur. Phys. J. D 22 (2003) 53.

[30] B. Franzke, H. Geissel, and G. Münzenberg, Mass Spectr. Reviews 27 (2008) 428. 
[31] W. Meng et al., Int. J. Mod. Phys. E (in press).

[32] Y. Yamaguchi et al., Nucl. Instr. and Meth. B 266 (2008) 4575.

[33] Yu. A. Litvinov et al., Nucl. Phys. A 756 (2005) 3.

[34] M. Hausmann et al., Nucl. Instr. and Meth. A 446 (2000) 569.

[35] J. Stadlmann et al., Phys. Lett. B 586 (2004) 27.

[36] J. Trötscher et al., Nucl. Instr. and Meth. B 70 (1992) 455

[37] B. Sun et al., Nucl. Phys. A 812 (2008) 1.

[38] R. K. Wallace and S. E. Woosley, Astrophys. J. Suppl. 45 (1981) 389.

[39] H. Schatz et al., Phys. Rep. 294 (1998) 167.

[40] C. Fröhlich et al., Phys. Rev. Lett. 96 (2006) 142502.

[41] J. Pruet et al., Astrophys. J. 644 (2006) 1028.

[42] H. Schatz et al., Phys. Rev. Lett. 86 (2001) 3471.

[43] H. Schatz, Int. J. Mass Spectrom. 251 (2006) 293.

[44] J. A. Clark et al., Phys. Rev. C 75 (2007) 032801(R).

[45] J. A. Clark et al., Phys. Rev. Lett. 92 (2004) 192501.

[46] P. Schury et al., Phys. Rev. C 75 (2007) 055801.

[47] D. Rodríguez et al., Phys. Rev. Lett. 93 (2004) 161104.

[48] G. Sikler et al., Nucl. Phys. A 763 (2005) 45.

[49] F. Herfurth et al., in preparation.

[50] G. Audi, A.H. Wapstra, and C. Thibault, Nucl. Phys. A 729 (2003) 337.

[51] A. Kankainen et al., Eur. Phys. J. A 29 (2006) 271.

[52] C. Weber et al., Phys. Rev. C 78 (2008) 054310.

[53] A. Martín et al., Eur. Phys. J. A 34 (2007) 341.

[54] V.-V. Elomaa et al., submitted to Eur. Phys. J. A (2008).

[55] M. Breitenfeldt et al., in preparation.

[56] M. Block, private communication.

[57] J. A. Clark et al., Eur. Phys. J. A. 25 S01 (2005) 629.

[58] J. Fallis et al., Phys. Rev. C 78 (2008) 022801(R).

[59] J. Fallis et al., these proceedings.

[60] J. L. Fisker, R. D. Hoffman, and J. Pruet (2007), arXiv:0711.1502.

[61] R. D. Hoffman, et al. (2008), arXiv:0801.1502.

[62] C. Mazzochi et al., Phys. Rev. Lett. 98 (2007) 212501.

[63] A. Kankainen et al., in preparation.

[64] J. J. Cowan, F.-K. Thielemann, and J. W. Truran, Phys. Rep. 208 (1991) 267.

[65] B. Pfeiffer, K.-L. Kratz, F.-K. Thielemann, and W. B. Walters, Nucl. Phys. A 693 (2001) 282.

[66] K.-L. Kratz et al., Ap. J. 403 (1993) 216.

[67] S. Wanajo et al., Ap. J. 606 (2004) 1057.

[68] J. W. Truran et al., Pubs. Ast. Soc. Pac. 114 (2002) 1293.

[69] A. Estradé et al., these proceedings.

[70] M. Matoš et al., Proceedings of the Zakopane Conference on Nuclear Physics 2008.

[71] B. Sun et al., Phys. Rev. C 78 (2008) 025806.

[72] W. Henning, Nucl. Phys. A 734 (2004) 654 and Nucl. Phys. A 805 (2008) 502c.

[73] T. Kubo et al., Nucl. Instr. and Meth. B 204 (2003) 97.

[74] http://www.er.doe.gov/np/program/FRIB.html. 